\title{
The Frequency of Low HER2 Expression in Breast Cancer and A Comparison of Prognosis Between Patients With HER2-Low and HER2-Negative Breast Cancer By HR Status
}

Nanae Horisawa ( $\nabla$ nhorisawa@aichi-cc.jp)

Aichi Cancer Center: Aichi-ken Gan Center

Yayoi Adachi

Aichi Cancer Center: Aichi-ken Gan Center

Daiki Takatsuka

Aichi Cancer Center: Aichi-ken Gan Center

Kazuki Nozawa

Aichi Cancer Center: Aichi-ken Gan Center

Yuka Endo

Aichi Cancer Center: Aichi-ken Gan Center

Yuri Ozaki

Aichi Cancer Center: Aichi-ken Gan Center

Kayoko Sugino

Aichi Cancer Center: Aichi-ken Gan Center

Ayumi Kataoka

Aichi Cancer Center: Aichi-ken Gan Center

Haruru Kotani

Aichi Cancer Center: Aichi-ken Gan Center

Akiyo Yoshimura

Aichi Cancer Center: Aichi-ken Gan Center

Masaya Hattori

Aichi Cancer Center: Aichi-ken Gan Center

Masataka Sawaki

Aichi Cancer Center: Aichi-ken Gan Center

Hiroji Iwata

Aichi Cancer Center: Aichi-ken Gan Center

Research Article 
Keywords: Low HER2 expression breast cancer, HER2-low, HER2-0, prognosis, trastuzumab deruxtecan

Posted Date: March 30th, 2021

DOI: https://doi.org/10.21203/rs.3.rs-298935/v1

License: (c) (1) This work is licensed under a Creative Commons Attribution 4.0 International License. Read Full License

Version of Record: A version of this preprint was published at Breast Cancer on October 7th, 2021. See the published version at https://doi.org/10.1007/s12282-021-01303-3. 


\section{Abstract}

\section{Purpose}

The DESTINY-Breast04 clinical trial is currently investigating whether trastuzumab deruxtecan (T-DXd) is effective in HER2-low as well as HER2-positive breast cancer. This highlights the interest in treatment strategies for patients with HER2-low breast cancer. The current study was therefore designed to determine the frequency of HER2-low among all breast cancers, and to compare the prognosis of HER2low patients with that of HER2-negative patients.

\section{Methods}

We retrospectively reviewed the biological data from 4,918 of 4,977 primary breast cancer patients who attended our institute. We quantified the overall frequency of breast cancer patients with a new HER2-low subtype that was defined by an immunohistochemistry score of $\mathrm{IHC} 1+$ or $\mathrm{IHC} 2+/ \mathrm{ISH}$. We then compared the clinical characteristics and prognosis of HER2-low patients with that of patients who did not have HER2 amplification (HER2-0).

\section{Results}

Low HER2 expression was found in 3169 (64.4\%) patients; 2860 (58.1\%) were HR-positive and 309(6.3\%) were HR-negative. Among HER2-0 patients, 681(13.9\%) were HR-positive and 157(3.2\%) were HRnegative. The HER2-0 group tended to have more poor prognostic factors than the HER2-low group, irrespective of HR status. There were no statistically significant differences between the prognosis of HER2-low and HER2-0 patients, regardless of HR status. However, patients in the HER2-low group tended to have better prognosis than those in the HER2-0 group.

\section{Conclusion}

HER2-low patients did not have a significantly different prognosis than HER2-0 patients, regardless of HR status. However, we should consider tailoring therapies for patients with HRE2-low early breast cancer according to their HR status.

\section{Introduction}

Approximately 15 to $20 \%$ of breast cancer patients have overexpression of human epidermal growth factor receptor 2 (HER2) [1, 2], and breast cancer patients with high levels of HER2 have a worse prognosis than those who are HER2-negative [1, 3-5]. However, the advent of humanized anti-HER2 monoclonal antibodies such as trastuzumab and pertuzumab has significantly improved the prognosis for patients with HER2-positive breast cancer [6-8]. Moreover, the EGFR and HER2 tyrosine kinase inhibitor lapatinib, has also been approved for use in HER2-positive breast cancer [9]. More recently, HER2 targeting antibody-drug conjugates (ADCs) including trastuzumab emtansine (T-DM1) and trastuzumab deruxtecan (T-DXd), have been developed in order to treat HER2-positive breast cancer [10,11]. 
American Society of Clinical Oncology (ASCO)/ College of American Pathologist (CAP) guidelines have been widely used to estimate the HER2 score in many countries. According to the guidelines, there are three categories of HER2 status that are based on immunohistochemistry (IHC) analysis, as follows. Patients are HER2-negative if the IHC score is 0 or $1+$, HER2-equivocal if the score is $2+$, and HER2positive if the score is $3+$. The HER2 status of patients with equivocal IHC scores are further examined using in situ hybridization (ISH). ISH-positive and ISH-negative tumors and then classified as HER2postive and -negative, respectively $[12,13]$. Several HER2 targeting therapies, such as trastuzumab, pertuzumab, lapatinib and T-DM1 have been specifically approved only for HER2-positive breast cancer as defined in the ASCO/CAP guidelines. These therapeutic options are therefore not available to patients with $\mathrm{IHC} 1+$ or $\mathrm{IHC} 2+/ \mathrm{ISH}$ - scores; even though they have slightly elevated HER2 levels they are categorized as HER2-negative $[12,14]$. However, it has recently been suggested that T-DXd may be effective not only in HER2-positive breast cancer, but also in HER2-low breast cancer, which would include patients with $\mathrm{IHC} 1+$ and $\mathrm{IHC} 2+/ \mathrm{ISH}$ - scores [15].

Given these observations, we suggest that it is critical to understand both the frequency and prognostic significance of HER2-low status in breast cancer. If, for example, HER2-low status was associated with a poorer prognosis than HER2-positive status, this would prompt a re-evaluation of the utility of anti-HER2 therapies in HER2-low patients. Although there is one report in which the prognoses of HER2-low and HER2-0 breast cancer were compared [16], further studies are required. We therefore carried out a retrospective analysis of HER2-low versus HER2-negative patients. The results of this analysis are described herein, and include stratification of cases based on hormone receptor (HR) status.

\section{Methods}

We retrospectively reviewed the data from 4,977 primary breast cancer patients who had received treatment at our institute between January 2005 and December 2015 (Fig. 1). The data collected included HER2 status, HR status, age, tumor size, the number of metastatic lymph nodes and histological grade from clinical records. We excluded patients for whom biological data were not available. We then investigated the proportion of patients that had low HER2 expression and compared the prognosis and background of the HER2-low group with the same parameters in the HER2-0 group; these data were stratified by HR status.

\section{Pathological assessment and definition of molecular subtypes}

Histopathological diagnoses of breast cancer were made by several pathologists at Aichi Cancer Center Hospital. HER2 score was determined by IHC; if the IHC score was equivocal, samples were subjected to ISH. The criteria of the Trastuzumab Pathology Group, which is referred to in the ASCO/ CAP guidelines, were used to determine the HER2 score. Tumors were defined as HR-positive if they yielded an Allred score of 3 or more for ER or PgR. We categorized new HER2 subtypes as follows. HER2-high if IHC3+ or IHC2+/ISH+, HER2-low if IHC 1+ or IHC 2+/ISH-, HER2-negative (HER2-0) if there was no HER2 staining. The categorization of new HER2 subtypes is shown in Table 1. 


\section{Statistical analysis}

The baseline characteristics were evaluated using Pearson's $\chi 2$ test. The endpoint of our study was comparison of 5-year overall survival (OS) and 5-year disease-free survival (DFS) between the HER2-0 group and the HER2-low group, according to HR status. DFS was defined as the time from the day of surgery to the day of recurrence or death from any cause. OS was defined as the time from the day of surgery to the death from any cause. Differences in five-year DFS and OS between HER2-low and HER2-0 patients and according to HR status were estimated using the log-rank test and the Kaplan-Meier method. A $p$ value less than 0.05 was considered statistically significant in our analysis. All data analysis was carried out using Stata Ver15.

\section{Results}

\section{Patients}

We obtained biological data from 4918 breast cancer patients (Fig. 1). In the new subtype categories, low HER2 expression was found in 3169 (64.4\%) of all breast cancer patients. There were 2860 HRpositive/HER2-low patients (58.1\%), and 309 HR-negative/HER2-low patients (6.3\%). There were 838 HER2-0 patients (17.1\%) overall; 681 were HR-positive (13.9\%) and 157 were HR-negative (3.2\%) (Table 2). The baseline characteristics of the HER2-low group and the HER2-0 group by HR status are shown in Table 3. Patients in the HR-negative/HER2-0 group tended to have larger tumors than those in the HRnegative/HER2-low group, whereas those in the HR-negative/HER2-low group had a higher frequency of in situ carcinoma. As observed in the HR-negative group, the HR-positive/HER2-0 group had larger tumors and worse historical grade $(\mathrm{HG})$ when compared with the HR-positive/HER2-low group. The frequency of ILC was higher in the HR-positive/HER2-low group than in the HR-positive/HER2-0 group. Regardess of HR status, there were no statistical differences between the HER2-low group and the HER2-0 group regarding lymph node metastasis, adjuvant chemotherapy or adjuvant endocrine therapy.

\section{DFS and OS}

The median follow-up time was 5.5 years. Five-year DFS in the HR-positive/HER2-low group and the HRpositive/HER2-0 groups was $91.6 \%$ and $90.1 \%$, respectively; these rates were not significantly different ( $p$ $=0.151$; Fig. 2-A). There was also no significant difference in 5-year OS between the HR-positive/HER2low group and the HR-positive/HER2-0 group ( $96.7 \%$ and $94.9 \%$, respectively, $\mathrm{p}=0.215$; Fig. 2-B).

HR-negative cases had a poorer overall prognosis than HR-positive cases. Specifically, 5-year DFS was $74 \%$ in the HR-negative/HER2-0 group and $78.7 \%$ in the HR-negative/HER2-low group; these values were not significantly different $(p=0.306 ;$ Fig2-C). A similar result was seen for 5 -year OS among HR-negative patients (Fig2-D).

\section{Discussion}


According to previous studies, between $40 \%$ and $50 \%$ of patients with breast cancer have tumors with low HER2 expression [15-17]. Here we observed a slightly higher proportion of HER-low cases (64\% of all breast cancers). The reason for the differences in frequency are currently unclear. However, one explanation is that there may be differences in the methods of quality control for the HER2 test, as we describe below. We also found that the overall incidences of HR-positive/HER2-low and HRnegative/HER2-low cases were $58.1 \%$ and $6.3 \%$, respectively.

There have been several reports regarding the prognosis of breast cancer patients with low HER2 expression. Ignatov et al. reported that patients with an intermediate HER2 score (defined as IHC 2+ and ISH-negative) had a worse prognosis than HER2-negative patients (defined as IHC 0 or 1+) breast cancer [18]. However, their study did not further stratify the patients based on HR status, which is an important parameter in the clinic. Francesco et al. were the first to compare the prognosis of HER2-low and HER2-0 by HR status, and found no statistically significant differences these two groups, regardless of HR status [16]. We suggest that it is more important to determine whether HER2-low or HER2-0 status is associated with a poorer prognosis in luminal or triple negative breast cancer. Our current study was designed to address this question, and the results suggest that HER2-low patients did not have a significantly different prognosis than HER2-0 patients, which is consistent with Francesco et al. However, we did find that the HER2-low group tended to have a better prognosis than the HER2-0 group regardless of HR status, although the differences were not statistically significant. We infer that this is because patients in the HER2-0 group tended to have more factors associated with poor prognosis than those in the HER2low group. Further studies are required to fully understand the prognostic significance of different levels of HER2 expression in breast cancer.

There are currently no approved guidelines that recommend anti-HER2 therapy for breast cancers with low HER2 expression. However, several studies have assessed anti-HER2 therapy in HER2-low breast cancer patients. The use of trastuzumab as adjuvant therapy in this setting did not improve DFS [19], nor did trastuzumab emtansine (T-DM1) improve DFS in metastatic breast cancer with low HER2 expression [20]. On the other hand, T-DXd may be effective for the treatment of breast cancer patients with low HER2 expression. T-DXd is a novel, HER2targeted ADC that is composed of a humanized monoclonal antibody attached by a cleavable peptide-based linker to a potent topoisomerase I inhibitor payload [21]. In the DESTINY-Breast 01 phase『study, T-DXd seemed to be effective in $44 \%$ patients with metastatic disease and low HER2 expression [21]. These results lead to the DESTINY-Breast04 phase III randomized trial, which is currently evaluating T-DXd for the treatment of HER2-low metastatic or advanced breast cancer (ClinicalTrials.gov identifier: NCT03734029). In our current study, there was no statistical difference between HER2-0 and HER2-low patients with regard to prognosis, irrespective of HR status. However, if TDXd and other anti-HER2 therapies are demonstrated to be effective in patients with HER2-low breast cancer, this should improve their overall prognosis. In particular, the prognosis of HR-negative/HER2-low breast cancer is poor, and novel therapeutic agents to treat patients in this group are clearly needed. HER2-low status may eventually be used as a predictive factor of the efficacy of novel therapeutics in early breast cancer, in much the same way that HER2-positive status is now used to stratify patients for TDXd treatment. 
The limitations of our current study are that it was carried out at a single institution, was retrospective and relied on inconsistent evaluation of HER2 scores. Moreover, different types of formalin solution can lead to different HER2 scores, which could contribute to inter-institutional discrepancies [22, 23]. In addition, the HER2 scoring scale was different in each study due to the continual updating of ASCO/CAP guidelines. However, our study is one of only a few that have determined the frequency and prognostic value of low HER2 expression in breast cancer in the context of of HR status.

\section{Conclusion}

There was no difference in prognosis between HER2-low and HER2-0 breast cancer patients regardless of HR status. However HER2-low patients, who will be candidates for new HER2-ADC drugs in the near future, accounted for over half of all early breast cancer patients. The therapeutic strategies for treatment of HER2-low early breast cancer patients should be considered in the context of HR status.

\section{Declarations}

\section{Acknowledgements}

The authors would like to thank all the patients who have cooperated in providing information at Aichi Cancer Center Hospital. We would also like to thank the doctors, nurses, and technical staf of our hospital for their daily support.

\section{Author contributions}

$\mathrm{NH}$ performed the planning study and the statistical analysis and drafted the manuscript. YA and HI were involved in the drafting and revision of the manuscript. MS, MH, AY, HK, AK, KS, YO, YE, DT and KN participated in information and data collection. All authors read and approved the fnal manuscript.

Funding: This research did not receive any specific grant from funding agencies in the public, commercial, or not-for-profit sectors.

Conflicts of interest: The authors declare that they have no conflict of interest.

Ethics approval: Our study adhered to the Declaration of Helsinki principles and was approved by the Institutional Review Board of Aichi Cancer Center Hospital. An opt-out approach was used, and was accessible to patients via or website, (https://www.pref.aichi.jp/cancer-center/cc/01gaiyo/rinri/2020-1319.pdf).

Consent to participate: Informed consent was obtained from all individual participants included in the study.

\section{References}


1. Slamon, D.J., et al., Human breast cancer: correlation of relapse and survival with amplification of the HER-2/neu oncogene. Science, 1987. 235(4785): p. 177-82.

2. Slamon, D.J., et al., Studies of the HER-2/neu proto-oncogene in human breast and ovarian cancer. Science, 1989. 244(4905): p. 707-12.

3. Andrulis, I.L., et al., neu/erbB-2 amplification identifies a poor-prognosis group of women with nodenegative breast cancer. Toronto Breast Cancer Study Group. J Clin Oncol, 1998. 16(4): p. 1340-9.

4. Seshadri, R., et al., Clinical significance of HER-2/neu oncogene amplification in primary breast cancer. The South Australian Breast Cancer Study Group. J Clin Oncol, 1993. 11(10): p. 1936-42.

5. Tiwari, R.K., et al., HER-2/neu amplification and overexpression in primary human breast cancer is associated with early metastasis. Anticancer Res, 1992. 12(2): p. 419-25.

6. Slamon, D.J., et al., Use of chemotherapy plus a monoclonal antibody against HER2 for metastatic breast cancer that overexpresses HER2. N Engl J Med, 2001. 344(11): p. 783-92.

7. Swain, S.M., et al., Pertuzumab, trastuzumab, and docetaxel in HER2-positive metastatic breast cancer. N Engl J Med, 2015. 372(8): p. 724-34.

8. Swain, S.M., et al., Pertuzumab, trastuzumab, and docetaxel for HER2-positive metastatic breast cancer (CLEOPATRA study): overall survival results from a randomised, double-blind, placebocontrolled, phase 3 study. Lancet Oncol, 2013. 14(6): p. 461-71.

9. Geyer, C.E., et al., Lapatinib plus capecitabine for HER2-positive advanced breast cancer. N Engl J Med, 2006. 355(26): p. 2733-43.

10. Baselga, J., et al., Pertuzumab plus trastuzumab plus docetaxel for metastatic breast cancer. N Engl J Med, 2012. 366(2): p. 109-19.

11. Verma, S., et al., Trastuzumab emtansine for HER2-positive advanced breast cancer. N Engl J Med, 2012. 367(19): p. 1783-91.

12. Wolff, A.C., et al., Human Epidermal Growth Factor Receptor 2 Testing in Breast Cancer: American Society of Clinical Oncology/College of American Pathologists Clinical Practice Guideline Focused Update. J Clin Oncol, 2018. 36(20): p. 2105-2122.

13. Farshid, G., et al., ASCO/CAP 2018 breast cancer HER2 testing guidelines: summary of pertinent recommendations for practice in Australia. Pathology, 2019. 51(4): p. 345-348.

14. Liu, Z.H., et al., Impact of the updated 2018 ASCO/CAP guidelines on HER2 FISH testing in invasive breast cancer: a retrospective study of HER2 fish results of 2233 cases. Breast Cancer Res Treat, 2019. 175(1): p. 51-57.

15. Modi, S., et al., Antitumor Activity and Safety of Trastuzumab Deruxtecan in Patients With HER2-LowExpressing Advanced Breast Cancer: Results From a Phase Ib Study. J Clin Oncol, 2020. 38(17): p. 1887-1896.

16. Schettini, F., et al., Clinical, pathological, and PAM50 gene expression features of HER2-low breast cancer. NPJ Breast Cancer, 2021. 7(1): p. 1. 
17. Giuliani, S., et al., In a cohort of breast cancer screened patients the proportion of HER2 positive cases is lower than that earlier reported and pathological characteristics differ between HER2 3+ and HER2 2+/Her2 amplified cases. Virchows Arch, 2016. 469(1): p. 45-50.

18. Ignatov, T., et al., Moderate level of HER2 expression and its prognostic significance in breast cancer with intermediate grade. Breast Cancer Res Treat, 2015. 151(2): p. 357-64.

19. Fehrenbacher, L., et al., NSABP B-47/NRG Oncology Phase III Randomized Trial Comparing Adjuvant Chemotherapy With or Without Trastuzumab in High-Risk Invasive Breast Cancer Negative for HER2 by FISH and With IHC 1+ or 2. J Clin Oncol, 2020. 38(5): p. 444-453.

20. Yazaki, S., et al., Lower response to trastuzumab emtansine in metastatic breast cancer patients with human epidermal growth factor receptor 2 immunohistochemistry score of 2 and fluorescence in situ hybridization positive compared with immunohistochemistry score of 3: a retrospective study.

Anticancer Drugs, 2020. 31(9): p. 973-978.

21. Modi, S., et al., Trastuzumab Deruxtecan in Previously Treated HER2-Positive Breast Cancer. N Engl J Med, 2020. 382(7): p. 610-621.

22. Wolff, A.C., et al., American Society of Clinical Oncology/College of American Pathologists guideline recommendations for human epidermal growth factor receptor 2 testing in breast cancer. Arch Pathol Lab Med, 2007. 131(1): p. 18-43.

23. Umemura, S., et al., What causes discrepancies in HER2 testing for breast cancer? A Japanese ring study in conjunction with the global standard. Am J Clin Pathol, 2008. 130(6): p. 883-91.

\section{Tables}

Table 1 The categorization of new HER2 subtypes

\begin{tabular}{|llllll|}
\hline \multicolumn{1}{|c|}{ IHC } & $3+$ & $2+$ & $1+$ & 0 \\
ISH & & & & \\
Positive & HER2-high & HER2-high & HER2-high & HER2-high \\
\cline { 1 - 1 } Negative & (ISH was not tested) & HER2-low & (ISH was not tested) & (ISH was not tested) \\
\cline { 3 - 5 } & & &
\end{tabular}

\section{Table 2 The frequency of breast cancer patients with new HER2-low subtypes}




\begin{tabular}{|c|c|c|c|c|}
\hline & $\begin{array}{l}\text { HER2-high } \\
\text { (IHC3+, } \\
\text { IHC2+/ISH+) } \\
(\%)\end{array}$ & $\begin{array}{l}\text { HER2-low (IHC1+, } \\
\text { IHC2+ /ISH-) } \\
\text { (\%) }\end{array}$ & $\begin{array}{l}\text { HER2-0 } \\
\text { (\%) }\end{array}$ & $\begin{array}{l}\text { Total } \\
(\%)\end{array}$ \\
\hline $\mathrm{HR}+$ & $508(10.3)$ & $2860(58.1)$ & 681(13.9) & $4049(82.3)$ \\
\hline HRD & $403(8.2)$ & $309(6.3)$ & $157(3.2)$ & $869(17.7)$ \\
\hline Total & $911(18.5)$ & $3169(64.4)$ & $838(17.1)$ & $4918(100)$ \\
\hline
\end{tabular}

Frequencies shown are percentages of the overall total of patients in this study

Table 3 Baseline characteristics between the HER2-low group and the HER2-0 group by HR status 


\begin{tabular}{|c|c|c|c|c|c|c|}
\hline & \multicolumn{3}{|l|}{ HR-positive } & \multicolumn{3}{|c|}{ HR-negative } \\
\hline & HER2-low & HER2-0 & $P$ & HER2-low & HER2-0 & $P$ \\
\hline & $\nabla=2860(\%)$ & $\mathrm{n}=681(\%)$ & & $\nabla=309(\%)$ & $\triangle=157(\%)$ & \\
\hline Age & & & $<0.001$ & & & 0.06 \\
\hline$<65$ & $2262(79.1)$ & $496(72.8)$ & & 219(71.0) & $124(79.0)$ & \\
\hline$\geqq 65$ & $598(21.0)$ & $185(27.2)$ & & $90(29.1)$ & $33(21.0)$ & \\
\hline $\mathrm{T}$ & & & 0.04 & & & 0.002 \\
\hline Tis & 483(16.9) & $101(14.8)$ & & $66(21.4)$ & 15(9.6) & \\
\hline$<2 \mathrm{~cm}$ & 1796(62.8) & $414(60.8)$ & & $139(45.0)$ & $94(59.9)$ & \\
\hline $2 \sim 5 \mathrm{~cm}$ & $501(17.5)$ & $136(20.0)$ & & $82(26.5)$ & 31(19.8) & \\
\hline$>5$ & $49(1.7)$ & $22(3.2)$ & & $17(5.5)$ & $15(9.6)$ & \\
\hline unknown & $31(1.1)$ & $8(1.2)$ & & $5(1.6)$ & $2(1.3)$ & \\
\hline$N$ & & & 0.25 & & & 0.95 \\
\hline 0 & 1764(61.7) & $431(63.3)$ & & 198(64.1) & $104(66.2)$ & \\
\hline $1-3$ & $521(18.2)$ & 109(16.0) & & $56(18.1)$ & $27(17.2)$ & \\
\hline$\nabla 4$ & $227(7.9)$ & $46(6.8)$ & & $30(9.7)$ & $13(8.3)$ & \\
\hline unknown & $348(12.2)$ & $95(14.0)$ & & $25(8.1)$ & $13(8.3)$ & \\
\hline HG & & & $<0.001$ & & & 0.05 \\
\hline 1 & $943(33.0)$ & $273(40.1)$ & & $19(6.2)$ & $3(1.9)$ & \\
\hline 2 & $1290(45.1)$ & 268(39.4) & & $64(20.7)$ & $30(19.1)$ & \\
\hline 3 & $319(11.2)$ & 91(13.4) & & $200(64.7)$ & $117(74.5)$ & \\
\hline Unknown & $308(10.8)$ & $49(7.2)$ & & $26(8.4)$ & $7(4.5)$ & \\
\hline Histological type ${ }^{* \mathbb{Z}}$ & & & $<0.001$ & & & 0.005 \\
\hline IDC & 2083(72.8) & $472(69.3)$ & & $226(73.1)$ & $130(82.8)$ & \\
\hline ILC & 192(6.7) & $31(4.6)$ & & $9(2.9)$ & $4(2.6)$ & \\
\hline Other & $82(2.9)$ & 71(10.4) & & $26(8.4)$ & $17(10.8)$ & \\
\hline Chemotherapy & & & 0.193 & & & 0.213 \\
\hline Neoadjuvant Chemotherapy & $136(4.8)$ & $43(6.3)$ & & $56(18.1)$ & $32(20.4)$ & \\
\hline
\end{tabular}




\begin{tabular}{|c|c|c|c|c|c|c|}
\hline Adjuvant Chemotherapy & $637(22.3)$ & 135(19.8) & & 154(49.8) & $82(52.2)$ & \\
\hline No chemo & 1983(69.3) & $474(69.6)$ & & $81(26.2)$ & $29(18.5)$ & \\
\hline Unknown & 104(3.6) & $29(4.3)$ & & $18(5.8)$ & $14(8.9)$ & \\
\hline Endocrine therapy & 1994(69.7) & $468(68.7)$ & 0.686 & - & - & \\
\hline No endocrine & $744(26.0)$ & $179(26.3)$ & & - & - & \\
\hline Unknown & $122(4.3)$ & $34(5.0)$ & & - & - & \\
\hline Anti-HER2 therapy & & & $<0.001$ & & & 0.790 \\
\hline Adjuvant anti-HER2 therapy & $13(0.5)$ & 0 & & $1(0.3)$ & $1(0.6)$ & \\
\hline No anti-HER2 & 2014(70.4) & $433(63.6)$ & & 179(57.9) & $87(55.4)$ & \\
\hline unknown & $833(29.1)$ & $248(36.4)$ & & $129(41.8)$ & $69(44.0)$ & \\
\hline
\end{tabular}

※1 excluding in situ carcinoma and a histological sample that could not be categorized

\section{Figures}

\section{Primary breast cancer patients who}

\section{underwent surgery from 2005 to 2015}

$$
\mathrm{n}=59
$$

\section{Biological data was identified}

\section{$(n=4918)$}

\section{Figure 1}

Study design 

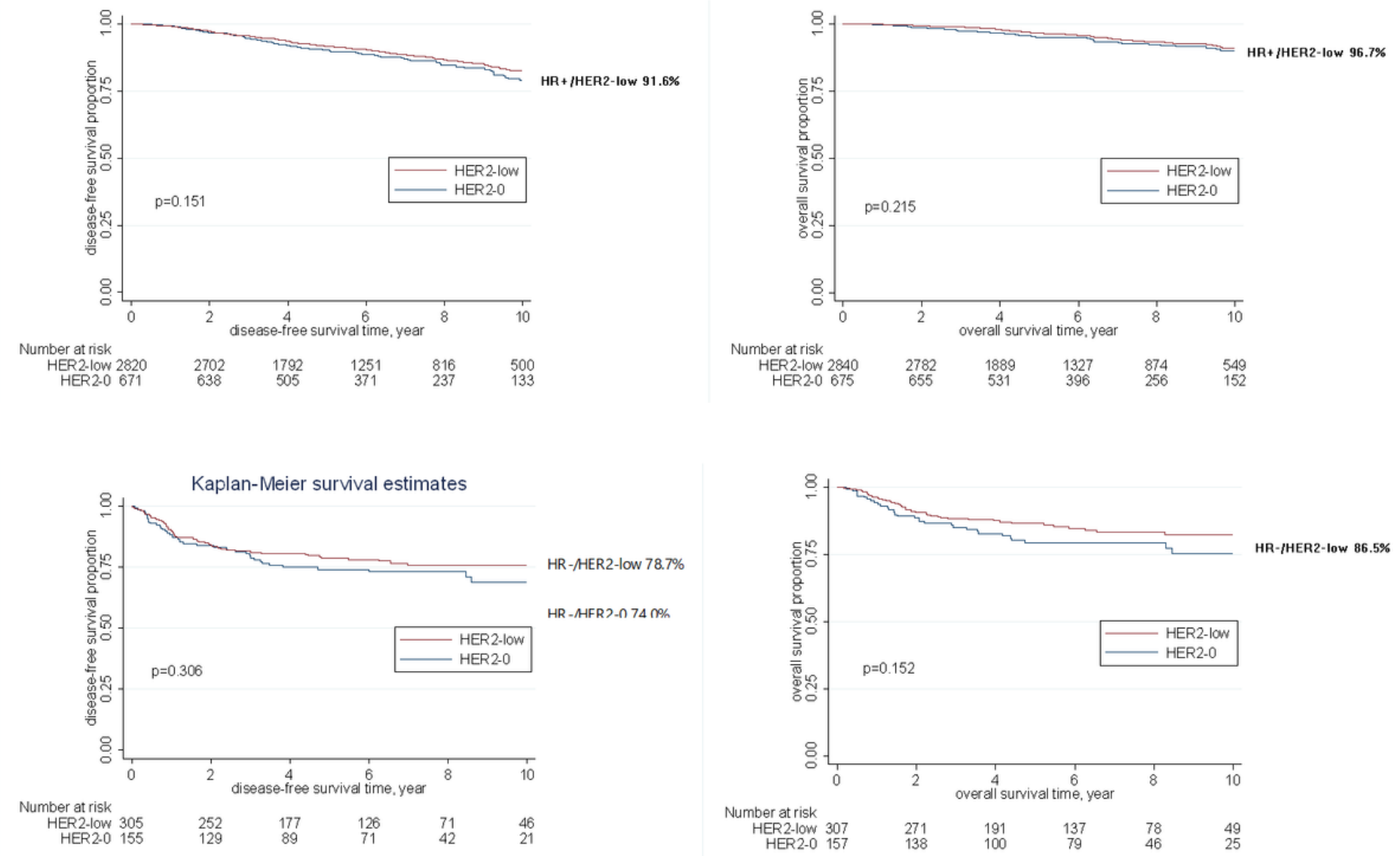

Figure 2

DFS and OS in HER2-0 and HER2-low patients according to HR status. Kaplan-Meier curves of DFS and OS are shown. DFS and OS of the HER2-low group vs the HER2-0 group in HR-positive patients are shown in A and B. DFS and OS of the HER2-low group vs the HER2-0 group in HR-negative patients are shown in $C$ and D. The $p$-values for log-rank tests are reported in each Figure panel. A. DFS in HR-positive patients B. OS in HR-positive patients C. DFS in HR-negative patients D. OS in HR-negative patients 